INPLASY

PROTOCOL

To cite: Pentapati et al.

Prevalence of Carpal Tunnel syndrome among dental health care providers -systematic review protocol. Inplasy protocol 202210084. doi: 10.37766/inplasy2022.1.0084

Received: 16 January 2022

Published: 16 January 2022

Corresponding author:

Kalyana Pentapati

drkalyan81@gmail.com

Author Affiliation:

Manipal Academy of Higher

Education, Manipal.

Support: Nil.

Review Stage at time of this submission: Preliminary searches.

Conflicts of interest:

None declared.

\section{Prevalence of Carpal Tunnel syndrome among dental health care providers -systematic review protocol}

Pentapati, K1; Chenna, D2; Kumar, M3; Madi, M4; Kumar, VS55.

Review question / Objective: What is the prevalence of Carpal Tunnel syndrome among dental health care providers?

Condition being studied: Carpal tunnel syndrome is median nerve peripheral neuropathy which causes paresthesia, pain, and numbness in territory of median nerve (thumb, index, middle, and lateral half of the ring finger).

Information sources: Pubmed, SCOPUS, EMBASE, CINAHL, Web of Sciences, Dentistry and Oral Science Source from inception to January 1st 2022.

INPLASY registration number: This protocol was registered with the International Platform of Registered Systematic Review and Meta-Analysis Protocols (INPLASY) on 16 January 2022 and was last updated on 16 January 2022 (registration number INPLASY202210084).

\section{INTRODUCTION}

Review question / Objective: What is the prevalence of Carpal Tunnel syndrome among dental health care providers?

Rationale: CTS is the most well-known and common form median nerve entrapment and accounts for $90 \%$ of all entrapment neuropathies. The prevalence of CTS among dental health care workers is higher due to repetitive bending movements of wrist, ulnar deviation of hand and repetitive pressure on palm. The prevalence of CTS is higher among dental health care providers than others. However, pooled prevalence of CTS was not reported among dental health care providers. Preventive training 
programs can be incorporated for reducing the prevalence of CTS.

Condition being studied: Carpal tunnel syndrome is median nerve peripheral neuropathy which causes paresthesia, pain, and numbness in territory of median nerve (thumb, index, middle, and lateral half of the ring finger).

\section{METHODS}

Search strategy: Pubmed, SCOPUS, EMBASE, CINAHL, Web of Sciences, Dentistry and Oral Science Source would be searched. A combination of keywords "dentist OR dental student OR dental auxiliary OR dental hygienist OR dental personnel AND carpal tunnel syndrome or carpal tunnel or medial nerve entrapment or CTS" would be used with appropriate filters for the above databases.

Participant or population: dentist OR dental student OR dental auxiliary OR dental hygienist OR dental personnel.

Intervention: Not applicable.

Comparator: Not applicable.

Study designs to be included: Observational studies such as crosssectional studies, case-control studies, cohort prospective studies and cohort retrospective studies will be included.

Eligibility criteria: Observational studies on dental health care personnel will be included. Only papers that are published in English language will be included. Studies published as letters, commentaries, short reports will be excluded.

Information sources: Pubmed, SCOPUS, EMBASE, CINAHL, Web of Sciences, Dentistry and Oral Science Source from inception to January 1st 2022.

Main outcome(s): Prevalence of carpal tunnel syndrome among dental health care providers.

Additional outcome(s): Side-specific prevalence if applicable, prevalence as per sex, geographic distribution, type of dental health care provider (dentists, dental auxiliary, dental students).

Data management: Search results would be added to Rayyan. Two trained review authors will be doing title and abstract screening followed by full-text screening independently. The data extraction would be done in a special proforma by two review authors independently.

Quality assessment / Risk of bias analysis: Risk of bias will be done with the use questionnaire developed by Hoy et al for prevalence studies by two review authors independently. Based on this questionnaire, studies will be identified as low, moderate or high risk.

Strategy of data synthesis: If a sufficient number of studies are available, data will be subjected to a meta-analysis of prevalence estimates using Open Meta software.

Subgroup analysis: Sub-group analysis would be done based on the sex, Sitespecific prevalence, Geographic distribution, type of dental health care workers, risk of bias.

Sensitivity analysis: Leave-one out metaanalysis would be performed

Language: English.

Country(ies) involved: India.

Keywords: carpal tunnel syndrome, dental health care providers, prevalence.

Dissemination plans: We intend to publish the results after completing the review.

Contributions of each author:

Author 1 - Kalyana Pentapati - Concept, search, analysis, initial and final draft.

Email: drkalyan81@gmail.com

Author 2 - Deepika Chenna - full-text screening, risk of bias assessment, the initial draft.

Email: deepu.kkd@gmail.com 
Author 3 - Mathangi Kumar - Title and abstract screening, data extraction, the final draft.

Email: madhukumar4u@gmail.com

Author 4 - Medhini Madi - full-text screening, data extraction, the final draft.

Email: medhini.madi@gmail.com

Author 5 - Vijay $S$ Kumar - Title and abstract screening, full- text screening, the initial draft.

Email: vijaytvpm24@gmail.com 